\title{
Dipole Moments and their Interpretation
}

$\mathrm{F}^{\mathrm{B}}$ WW branches of physical chemistry can show a more rapid development than the study of dipole moments. The theoretical work of Debye goes back to a discussion of the Mosotti-Clausius equation in 1912, but the experimental work really begins with Zahn's measurements on gases in 1924 and the work of Smyth, Williams and others on solutions a few years later. To-day, values of the dipole moment have been determined for more than a thousand substances, and measurements of dielectric properties are applied to such varied topics as the determination of valency angles, the size of colloid molecules, and the order of the boiling points of isomeric substances. It is not surprising therefore that the discussion on "The Determination and Interpretation of Dipole Moments" held by the Faraday Society at Oxford on April 12-14 attracted a large number of British and foreign workers in this field. The outstanding figure was, of course, that of Debye ; those who attended the meeting will long remember his shrewd comments on every paper, his genial smile and his cigar which served so aptly as a model of a dipole.

It is only possible to mention a few of the many papers which were read. The first group was concerned with the determination of electric moments of molecules. It seems to be generally agreed that atomic polarisations are small and are rarely greater than 5 c.c. Anomalous dipole moments can now no longer be ascribed to large values of $P_{A}$. On the other hand, the work of Horst Müller, Jenkins and others shows clearly that the magnitude of the dipole moment deduced from measurements in solution is influenced by the dielectric constant of the solvent. A number of formulæ connecting measured polarisation and dielectric constant were discussed, but much more work on vapours as well as on solutions is needed before the solvent effect can be accurately determined.

Debye's opening paper contained an account of novel work by Martin on dielectric losses in dilute solutions of a polar substance in a non-polar solvent. Following Malsch, the thermal expansion of the solution was used as a delicate method of measuring the heat developed by absorption of electrical energy. Non-polar substances gave negligible heating but polar substances showed a marked effect. Thus with 0.1 molecular solutions of orthodichlorobenzene $\left(\mu=2 \cdot 25 \mathrm{D}^{*}\right)$ and paradichlorobenzene $(\mu=0)$, the relative heating effects were in the ratio $310: 1$. Theory indicates that the effect should be proportional to the square of the dipole moment $\mu$, and from the measured energy absorption the relaxation time of the polar molecule can be computed. In accordance with elementary theory, this is found to be of the order of $10^{-12}$ seconds for solutions in solvents of low viscosity, such as benzene. The elementary theory assumes spherical molecules and Stokes's law, and the experimental data diverge considerably from the predicted results. It seems probable that further investigations along these lines may give information about the shapes of molecules.

Determinations of relaxation time from measurements of anomalous dispersion at high radio frequencies were discussed in the papers contributed

$* \mathrm{D}$ is the Debye unit, $10^{-18}$ E.s.u. c.G.s. by Williams and by Girard. The latter finds anomalies in the shape of the dispersion curve for glycerol which he ascribes to the presence of two species of molecules, namely, normal and associated molecules. Girard also notes some curious empirical relations between the dielectric properties of hydroxyl compounds. Thus for the normal aliphatic alcohols from propyl alcohol upwards, the product of the molecular wejght and the dielectric constant at $20^{\circ} \mathrm{C}$. is nearly constant.

Another group of papers was concerned with the problem of free rotation. There is, of course, a mass of chemical evidence in favour of free rotation about a single bond. More accurately, it should be said that if isomerides exist which differ in structure merely by the relative angular orientation of groups about a single bond, then these isomerides are so readily interconvertible that they cannot be isolated. Dipole moments give a good deal more information about such rotations. Thus the existence of a dipole moment for hydroquinone dimethyl ether is explained by the rotation of the $-\mathrm{OMe}$ groups about the $\mathrm{C}-\mathrm{O}$ bond. Williams and others have discussed molecules of the type of ethylene dichloride and distinguished three extreme cases : $(a)$ the repulsions between the chlorine atoms fixes them in the trans position giving $\mu=0 ;(b)$ the chlorine atoms are fixed in the cis position and $\mu=3 \cdot 6 \mathrm{D}$; (c) the $\mathrm{CH}_{2} \mathrm{Cl}$ groups rotate freely about the $\mathrm{C}-\mathrm{C}$ axis with all orientations equally probable and $\mu=2 \cdot 5 \mathrm{D}$. Zahn has measured the dipole moment of ethylene dichloride in the vapour state and finds that it varies with temperature from $1 \cdot 12 \mathrm{D}$ at $32^{\circ} \mathrm{C}$. to $1 \cdot 54 \mathrm{D}$ at $281^{\circ} \mathrm{C}$. This is interpreted as indicating that the trans position is the most stable, and that increasing thermal vibrations give oscillations from this position. The problem is, however, one which deals with phenomena on the atomic scale and can only be solved satisfactorily by the methods of wave mechanics. An illuminating discussion of the restriction of free rotation in molecules of the type $\mathrm{XCH}_{2} \mathrm{CH}_{2} \mathrm{X}$ was given by Lennard Jones. From suitable molecular models a wave equation was set up and an equation obtained which accounts quantitatively for the change of dipole moment with temperature.

Another interesting quantum discussion of a similar problem was given by Penney and Sutherland on the structure of hydrogen peroxide and hydrazine. These molecules give unexpectedly large dipole moments, which have been ascribed to free rotation. An examination of the problem by quantum mechanical methods shows that the main forces determining the structure of the molecule are not the electrostatic repulsion between the terminal hydrogen atoms but the interaction between the electron clouds associated with the oxygen atoms in $\mathrm{HOOH}$ and the nitrogen atoms in $\mathrm{H}_{2} \mathrm{NNH}_{2}$. The most stable configuration for hydrogen peroxide is a skew one in which the planes through the $\mathrm{O}-\mathrm{O}$ axis and the $\mathrm{H}$ atoms are nearly at right angles. In other words, the most stable position is about halfway between the cis and trans positions. A similar structure is found for hydrazine; for both sub. stances the observed dipole moments are in fair agreement with the values calculated for the skew configuration. 
A group of papers was devoted to the discussion of resonance phenomena. Here one is concerned primarily with the rapid oscillation of electrons between different positions in the molecule, and not with the movements of nuclei as in the free rotation problem. The problem of the structure of the organic azides was discussed by Sidgwick. The azide ion has undoubtedly the linear structure $\mathrm{N} \leftrightarrows \mathrm{N} \rightrightarrows \mathrm{N}$; for the covalent organic azides three structures are possible as shown below. Parachor and volatility favour I, whilst chemical reactions, electron diffraction, and recent crystal structure measurements reported by Bernal indicate a linear structure, II or III. The dipole moments of a number of azides give for the moment of the $\mathrm{Ph}-\mathrm{N}_{3}$ group about $1 \cdot 5 \mathrm{D}$. This is scarcely larger than the moment of the $\mathrm{Ph}-\mathrm{N}$ group, so that the links in the $\mathrm{N}_{3}$ group contribute very little to the moment. II and III should give

$$
R-N \|_{N}^{N} \underset{I I}{\longrightarrow}
$$$$
\begin{gathered}
\mathrm{R}-\mathrm{N} \leftarrow \mathrm{N} \equiv \mathrm{N} \\
\leftarrow
\end{gathered}
$$

III

large contributions in the senses indicated by the arrows below the formulæ, so the dipole moments seem to favour structure I. This is, however, quite incompatible with the electron diffraction and crystal measurements.

The low moment cannot be reconciled with a linear arrangement of the nitrogen atoms by postulating tautomerism in the chemical sense of the word between forms II and III. If the time of interchange between the two forms is less than the relaxation time $\left(c .10^{-12} \mathrm{sec}\right.$.), then each form would orient under the influence of the applied field and the observed moment would be large, although the two forms have moments of opposite sign. Quantum mechanical resonance between the two forms with an interchange time of the order of $10^{-15} \mathrm{sec}$. would account for the observed moment. For this to occur it is necessary for the two forms to have equal or nearly energies. Sidgwick has computed the heats of formation of the $\mathrm{N}_{3}$ group making certain plausible assumptions with the following results :

$$
\text { I, } 170 \mathrm{k} \text {. cal. II, } 191 \mathrm{k} \text {. cal. III, } 180 \mathrm{k} \text {. cal. }
$$

The observed value calculated from the heats of combustion of organic azides is $211 \mathrm{k}$. cal. This seems to exclude $\mathbf{I}$ and is compatible with resonance between II and III, since resonance increases the stability of the molecule and would increase the heat of formation.

The vector addition of dipole moments and the calculation of valency angles were considered in another group of papers. The chief difficulty met with in this field is the uncertain magnitude of the interaction between two dipoles in a molecule or between a dipole and the polarisable part of the same molecule. Hampson described a method of eliminating such errors considering the moments of a series of compounds, for example :
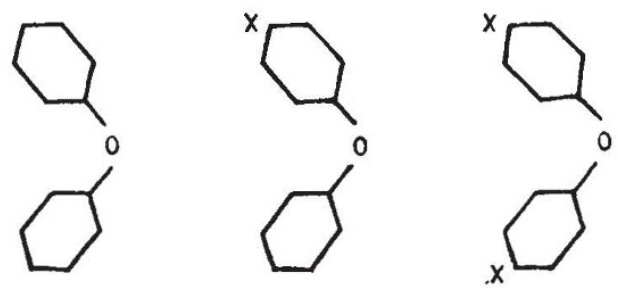

where $\mathrm{X}$ is a group of known moment. From the three measured moments it is possible to fix upper and lower limits for the valency angle and to estimate the magnitude of the perturbing induced dipole along the $\mathrm{X}-\mathrm{O}$ axis. For the oxygen valency angle consistent values of about $130^{\circ}$ were found; the induced dipole was small when $\mathrm{X}$ was $\mathrm{Cl}$ but when $\mathrm{X}$ was $\mathrm{NO}_{2}$ amounted to half a Debye unit.

Finally, mention must be made of the remarkable results found by Hassel for certain cyclohexane derivatives. $1: 4$ dichloro-, dibromo-, and di-iodo cyclohexane were found to have zero moment in solution in benzene. This result is not easily reconciled with the usual view that cyclohexane derivatives consist of a tautomeric mixture of two strainless forms with a non-planar configuration.

S. S.

\section{University Statistics of Great Britain}

$\mathrm{U}^{\mathrm{N}}$ NIVERSITY statistics of Great Britain for the year 1932-33, recently issued by the University Grants Committee*, show that the attendance of students has been well maintained and university finances have a healthy appearance. It would seem that the measures taken to temper the effect on universities in Great Britain of the inclement economic weather prevailing in the past few years have achieved their object. The enrolment of full-time students has been gradually rising since 1924-25 and showed last year a further increase of $1,255-1,267$ more men and 12 fewer women. of the aggregate total of 50,155 , five per cent were engaged in research and other advanced work, seventy-seven per cent were reading for a first degree and eighteen per cent for a diploma. Part-time students numbered 13,960 , of whom rather more than

* University Grants Committee. Returns from Universities and University Colleges in receipt of Treasury Grant, Academic Year 1932-33. Pp. 26. (London: H.M. Stationery Office, 1934.) 18. $3 d$. net. half were not pursuing regular courses leading to a degree or diploma.

A regional distribution of the full-time students gives to: London, 24 per cent ; Oxford and Cambridge, 21 per cent; provincial universities of the Midlands and northern England, $21 \frac{1}{2}$ per cent; Reading, Southampton, Exeter and Bristol, 5 per cent; Wales, 62 $\frac{2}{3}$ per cent; and Scotland, 22 per cent.

Students from abroad, that is, from homes outside the British Isles, numbered 5,870-about one tenth of the total number-considerably more than half of them being from countries within the British Empire: these show, however, a tendency to decrease in proportion to those from foreign countries. The following table gives particulars in respect of universities where such visitors from abroad were most numerous, showing (1) full-time students from places outside the British Isles but within the Empire, (2) those from foreign countries, and (3) the 